ÉGYPTE monde arabe

\section{Égypte/Monde arabe}

12-13 | 1993

Une économie en transition

\title{
Tenter de comprendre les causes de la discorde
}

Al-Ahrâm, 27 mai 1992

\section{Rafiq Habib}

Traducteur : Mona Akouri

\section{(2) OpenEdition}

\section{Journals}

Édition électronique

URL : https://journals.openedition.org/ema/1280

DOI : 10.4000/ema. 1280

ISSN : 2090-7273

\section{Éditeur}

CEDEJ - Centre d'études et de documentation économiques juridiques et sociales

\section{Édition imprimée}

Date de publication : 31 mars 1993

Pagination : 254-258

ISSN : 1110-5097

\section{Référence électronique}

Rafiq Habib, «Tenter de comprendre les causes de la discorde », Égypte/Monde arabe [En ligne], 12-13। 1993, mis en ligne le 08 juillet 2008, consulté le 07 juillet 2022. URL : http://journals.openedition.org/ ema/1280 ; DOl : https://doi.org/10.4000/ema.1280

Ce document a été généré automatiquement le 7 juillet 2022.

Tous droits réservés 


\section{Tenter de comprendre les causes de la discorde}

Al-Ahrâm, 27 mai 1992

Rafiq Habib

Traduction : Mona Akouri

\section{NOTE DE L'ÉDITEUR}

Le Dr. Rafiq Habib est de rite anglican ; il est l'auteur de plusieurs ouvrages sur les mouvements politico-religieux en Égypte, qui renvoient dos-à-dos les fondamentalistes coptes et musulmans. Il est par ailleurs très critique vis-à-vis de l'Église copteorthodoxe, plus particulièrement le patriarche Chenouda III, dont il estime qu'il joue un rôle politique explicite et mobilise à cet effet la communauté copte.

1 Dans le quotidien Al-Ahrâm en date du 12 mai 1992, le professeur Fahmi Huwaydi faisait paraitre un "rapport $»^{1}$ sur les désordres survenus en Amérique, révélant que les troubles de Los Angeles n'étaient que le fruit d'un «complot fomenté par l'Organisation fondamentaliste islamique internationale, complot visant à détruire la civilisation occidentale, Amérique d'abord, Europe ensuite ». Les lecteurs ayant montré une tendance manifeste à accréditer cette version, M. Huwaydi s'est expliqué, le 19 mai, dans un nouvel article intitulé : «L'interprétation fondamentaliste de l'Histoire ». Il me semble que ces deux articles expriment bien la substance du message visé, à savoir la manière de traiter les crises auxquelles la société se trouve en butte.

2 La «manière arabe» consiste généralement en la découverte d'une "minorité dévoyée ", d'un "groupe fanatique ", et l'histoire commence et finit par la localisation de ladite minorité puis par sa liquidation, comme si cette minorité venait d'une autre planète et avait atterri au sein de la société. Personne ne se demande pourquoi cette minorité est apparue, pourquoi la colère est son mode d'expression privilégié, selon quels critères elle choisit sa victime... En d'autres termes, nous nous interrogeons moins sur le problème lui-même que sur le coupable. 
3 La « manière » américaine procède autrement : les médias américains ont parlé, eux, du racisme des Blancs, de la pauvreté rampante, de la révolte des affamés; ils ont critiqué les erreurs du régime, l'ont désigné comme responsable pour avoir négligé des problèmes internes devenus explosifs. Il ne s'agit ni de démocratie ni de liberté d'opinion. La signification de tout cela est bien plus importante et révèle un comportement conscient à l'égard des crises que peut affronter une société, la crainte éprouvée pour le système dominant pour la civilisation/culture dominante; c'est l'expression du désir du système lui-même de se renouveler, d'émerger de ses crises, de durer...

4 La façon de réagir des Américains leur a permis de désamorcer la colère, de sortir du cercle vicieux de la violence ; le régime s'est même empressé de reconnaitre ses torts au lieu de les nier et d'accumuler les erreurs. Si nous laissons de côté les expressions telles que "dissensions confessionnelles ", "groupes dissimulés derrière le masque de la religion" ou d'autres encore, qui alimentent les complaintes nationales aux lendemains des crises, nous verrons que les médias américains ont abordé plusieurs points importants :

1. l'incident a pour origine une décision judiciaire qui a réveillé dans l'esprit de certains le sentiment d'oppression qui s'y trouvait enfoui ;

2. une certaine catégorie de la population s'adonne à des actes de violence dénoncés par la société :

3. cette catégorie sociale est une victime de la société : cette dernière doit mettre un terme à l'injustice qui la frappe, et la victime renoncer à la violence ;

4. la majorité a opprimé cette catégorie sociale et s'est montrée raciste à son égard ;

5. le régime a négligé le problème de la pauvreté jusqu'à ce qu'il ait pris de graves proportions et se soit transformé en un courant dévastateur, etc.

5 Peut-on partir des mêmes éléments pour expliquer les incidents survenus à Dayrout, ou ceux d'Abu Qorqas, Beni Soueif, Minya, Imbaba, al-Zawiya al-Hamra... qui les ont précédés? De prime abord, il semble que ces éléments soient sans rapport avec ce qui s'est passé en Égypte; cependant, en allant davantage au fond des choses, il devient évident que nous ignorons ce qui s'est exactement passé, que seuls les détails de moindre importance ont marqué notre mémoire. Les points précités sont des interrogations auxquelles nous n'avons même pas tenté de répondre: car nous ne voulons pas nous avouer qu'il est important d'interroger pour comprendre; nous nous contentons d'abandonner l'affaire aux services de sécurité, leur laissant le loisir de se mettre à la recherche de la "minorité dévoyée ». Nous ne nous sommes pas demandé ce qui se passerait si cette minorité se transformait en majorité... Mais qui ne sait pas questionner ne saurait obtenir de réponse!

6 Tout a commencé avec les actes de violence perpétrés au cours de ces dernières années, actes que l'on a attribués aux groupes islamistes militants, à l'organisation Al-Jihadi à d'autres. Les pratiques violentes de ces organisations étaient dirigées contre le pouvoir, les forces de sécurité, les Frères musulmans, contre certains autres groupes islamiques même, contre les coptes, les musulmans. Dans la mesure où ces groupes s'en sont pris à plus d'une catégorie de la population, on ne peut plus parler de dissensions confessionnelles, sinon ces actions auraient pris les coptes pour cible unique. Il ne peut non plus s'agir de visées sur le pouvoir car en ce cas, ce dernier aurait aussi constitué la cible unique. 
7 Il vaudrait donc mieux avancer que les actions violentes ne sont que l'expression de la révolte d'une catégorie opprimée, c'est-à-dire l'expression de la victime qui n'a trouvé de justicier qu'elle-même. Quant au sujet agressé, c'est-à-dire la victime de l'incident et non celle de la conjoncture -, il représente le symbole supposé responsable de l'oppression subie. Je vais me hâter d'éclaircir ces propos avant que la défiance n'ait raison du lecteur et qu'il s'imagine que je vois en les coptes l'oppresseur, et l'opprimé en les groupes islamiques. La question n'est pas aussi simple. Dans le contexte américain, les Noirs ont incendié les magasins des Coréens alors que l'oppression exercée à leur encontre vient des Blancs, des Anglo-saxons, et non des Coréens, parmi lesquels certains appartiennent aux couches défavorisées tandis que d'autres sont privilégiés; d'où ce nouveau constat: toute cible visée par la violence n'est pas forcément responsable, au premier degré, du sort de ceux qui la mettent en œuvre, et pourrait même être la dernière concernée. Mais dans le cas que nous venons de citer, la violence était dirigée contre des symboles économiques : ce qui brûlait, c'était des marchandises dont les Noirs pouvaient difficilement être acquéreurs. Ces derniers ressentent et vivent l'injustice, la pauvreté ; puis survient une décision judiciaire qui innocente quatre officiers accusés du passage à tabac d'un chauffeur noir : voici établie la preuve que l'oppression est la règle, qu'elle est légalisée et institutionnalisée, d'où l'explosion de colère; moins pour changer les choses que pour affirmer le droit de l'opprimé à faire entendre son cri.

8 Certains s'empresseront de conclure que le problème réside dans la pauvreté; cependant, pour les Noirs, le problème ne se réduit pas à la pauvreté. S'y mêlent l'impossibilité de vivre dans la dignité, les regards hautains et méprisants de ceux qui jouissent d'une situation meilleure et qui, sans doute, sont cause du problème. Dès lors, la crise pèse principalement sur une couche sociale qui agonise... Oui, qui agonise. La pauvreté n'est pas un facteur suffisant pour engendrer la violence; et même s'il en était ainsi dans les pays où règne cette dernière, l'Égypte, avec sa tendance traditionnelle à la conciliation, ne constitue guère un terrain propice.

9 La pauvreté signifie l'incapacité de vivre, la perte, par l'individu, de toute place dans l'existence. Imaginons une catégorie sociale privée d'espace, de rôle, de choix, de droit à l'existence, une catégorie rejetée, méprisée, marginalisée : qu'en adviendrait-il ? Car il s'agit d'une pauvreté à trois dimensions - économique, sociale, politique - et qui, de plus, frappe une couche sociale agonisante. Celle-ci manifeste sa colère, brandit la bannière de la violence et ne trouve que ses propres forces pour échapper à la mort sociale et renaître à l'existence ; elle ne peut opérer cette renaissance qu'en affrontant, d'une part, la société perçue comme l'oppresseur et, d'autre part, se proposer comme étant l'alternative la meilleure et le proclamer aussi haut que possible; lorsque l'oppression se fait fortement ressentir, cette catégorie opprimée s'appuie souvent sur un idéal nouveau ou tombé dans l'oubli, afin de confondre la société dont elle dénonce la corruption et faire valoir son propre mérite : elle lui déclare la guerre.

La religion n'apparait-elle pas ici comme une autre voie, un substitut? Si tout cela est vrai, comment expliquer le choix des cibles visées par les groupes islamiques? En ce qui concerne le pouvoir, il lui est fait grief de la détérioration de la situation économique ou sociale, quel que soit le rôle joué en la matière par la conjoncture politique; quant aux services de sécurité, ce sont eux qui empêchent les groupes révoltés d'accéder au pouvoir. Les attaques des islamistes extrémistes contre les Frères musulmans constituent une offensive dirigée contre le concurrent en présence sur la 
scène islamique, rival qui s'oppose au pouvoir sans mettre en cause sa légitimité, mais traite au contraire avec lui dans le cadre du système établi, légitimant du même coup ce système ; dès lors, ce concurrent est perçu comme celui qui aurait pour rôle d'étouffer la colère, de barrer la route à la révolte.

11 Enfin, les violences qui opposent les mouvements islamistes entre eux sont l'indice révélateur d'un démantèlement interne: il ne s'agit pas d'un débordement qui ne trouverait de bouc émissaire que dans sa propre sphère parce qu'il lui serait impossible de s'extérioriser. Lorsque la violence se retourne contre le musulman de la rue, on peut y voir l'affrontement de deux systèmes, l'un qui contrôle la société et l'autre qui tente de se substituer au premier. L'attaque signifie qu'en définitive, ce dernier est considéré comme responsable de la situation, ne serait-ce que par son silence.

12 Par ailleurs, en abordant la question des coptes, nous sommes amenés à toucher l'essence de ce qu'on a appelé «dissensions confessionnelles» : pourquoi, en effet, parler d'hostilité religieuse alors qu'il s'agit en réalité d'une révolte générale, la violence visant les coptes ne représentant ni le phénomène dans sa totalité ni son essence et n'en constituant qu'un aspect. Cet aspect est important, certes, et se présente comme suit : en tant que chrétiens, les coptes sont appréhendés à travers une grille de signes particuliers; on doute de leur position concernant l'islam, ou du moins l'islam politique préconisé par les groupes islamistes (et en ce qui concerne ce dernier, le doute devient certitude). D'où un fanatisme réciproque et une aversion entre adeptes de religions différentes. Il suffit à ces groupes islamistes de se percevoir comme objet $\mathrm{du}$ ressentiment des chrétiens pour ranger ces derniers au nombre des ennemis, des obstacles. Ajoutons à cela que les services de sécurité se sont trouvés investis de la mission de protéger les coptes et leurs églises. De cette manière, le pouvoir peut se servir des coptes comme bouc émissaire ; comme c'est le copte qui me côtoie dans la rue, il constitue l'ennemi le plus proche, la cible la plus facile d'accès, tel l'indicateur de police qui est tué parce qu'il est plus facile de l'atteindre, lui, que d'atteindre quelqu'un de plus haut placé, etc.

Notre analyse peut-elle constituer un type d'interprétation susceptible d'éveiller entendement et conscience?

\section{NOTES}

1. Fahmi Huwaydi s'était en réalité livré à une expérience: il avait fait paraître un faux rapport, qui lui avait été soi-disant 'remis sous le sceau de la confidentialité », par de hauts responsables américains, texte satirique attribuant les troubles de Los Angeles à des groupes islamistes américains (NDLR). 
INDEX

Mots-clés : Habib (Rafiq), terrorisme, violence, Coptes 